\title{
The Inheritance of Peripapillary Atrophy
}

\author{
Paul R. Healey, ${ }^{1}$ Paul Mitchell, ${ }^{1}$ Clare E. Gilbert, ${ }^{2}$ Anne J. Lee, ${ }^{1}$ Dongliang Ge, ${ }^{3}$ \\ Harold Snieder, ${ }^{3,4}$ Timothy D. Spector, ${ }^{4}$ and Christopher J. Hammond ${ }^{4,5}$
}

Purpose. To estimate the relative importance of genes and environment in peripapillary atrophy type beta ( $\beta$-PPA) in a classic twin study.

Methods. Female twin pairs $(n=506)$ aged 49 to 79 years were recruited from the St. Thomas' UK Adult Twin Registry. Peripapillary atrophy was identified from masked grading of stereoscopic optic disc photographs. Structural equation modeling was performed using Mx with polychoric correlations of $\beta$-PPA and refractive error (divided into deciles).

Results. $\beta$-PPA prevalence was $25.1 \%$ and did not vary with zygosity. Case-wise concordance for right eyes was $0.76(95 \%$ CI, 0.57-0.88) for monozygotic (MZ) and 0.37 (95\% CI, 0.15$0.56)$ for dizygotic (DZ) pairs. Multivariate modeling suggested additive genetic effects and individual environment, with no shared environment or dominant genetic effect. $\beta$-PPA heritability was 0.70 (95\% CI, 0.54-0.83), and spherical equivalent 0.88 (95\% CI, 0.85-0.91); age had no significant effect on variance. The genetic correlation between $\beta$-PPA and spherical equivalent was -0.21 . However, only $3 \%$ of the genetic variance of $\beta$-PPA was explained by genetic factors in common with refractive error, with $67 \%$ explained by specific genetic factors for $\beta$-PPA. Of the $30 \%$ of variance explained by unique environmental factors, only $3 \%$ was explained by these factors in common with environmental factors involved in refractive error.

Conclusions. The presence of $\beta$-PPA, a frequent ocular finding known to be associated with open-angle glaucoma, appears to be under strong genetic control, with only a small amount of this genetic effect shared with genes involved in myopia. (Invest Ophthalmol Vis Sci. 2007;48:2529-2534) DOI: 10.1167/iovs.06-0714

C horioretinal atrophy surrounding the optic disc (known as either peri- or parapapillary atrophy) is a relatively common clinical finding. Jonas et al. ${ }^{1}$ have developed a classification of two zones: an area of visible sclera and large choroidal vessels, adjacent to the optic disc border, termed zone beta, and a more peripheral area of irregular hypo- and hyperpig-

From the ${ }^{1}$ Centre for Vision Research, University of Sydney, Australia; the ${ }^{4}$ Twin Research and Genetic Epidemiology Unit, St. Thomas' Hospital, London, United Kingdom; the ${ }^{2}$ London School of Hygiene and Tropical Medicine, London, United Kingdom; the ${ }^{3}$ Georgia Prevention Institute, Medical College of Georgia, Augusta; the ${ }^{5}$ West Kent Eye Centre, Bromley Hospitals NHS Trust, Orpington, United Kingdom.

Supported by the Ophthalmic Research Institute of Australia, the Wellcome Trust, London, and the Guide Dogs for the Blind Association, United Kingdom.

Submitted for publication June 27, 2006; revised December 28, 2006; accepted April 23, 2007.

Disclosure: P.R. Healey, None; P. Mitchell, None; C.E. Gilbert, None; A.J. Lee, None; D. Ge, None; H. Snieder, None; T.D. Spector, None; C.J. Hammond, None

The publication costs of this article were defrayed in part by page charge payment. This article must therefore be marked "advertisement" in accordance with 18 U.S.C. $\$ 1734$ solely to indicate this fact

Corresponding author: Paul R. Healey, Centre for Vision Research, University of Sydney, Westmead Hospital, Hawkesbury Road, Westmead, NSW, Australia, 2145; phealey@glaucoma.net.au. mentation, termed zone alpha. ${ }^{1}$ This classification into $\beta$ - and $\alpha$-peripapillary atrophy ( $\beta$-PPA and $\alpha$-PPA) has now been accepted into common use, with reports of an association with glaucoma made principally with $\beta$-PPA.

$\beta$-PPA atrophy has been reported to be associated with both the prevalence $\mathrm{e}^{2-7}$ and progression ${ }^{8-11}$ of open-angle glaucoma (OAG). The presence of $\beta$-PPA ${ }^{12}$ and its enlargement over time ${ }^{13}$ have been reported to precede and predict the onset of glaucoma in ocular hypertensive eyes.

In examining the heritability of OAG, it is important to examine the inheritance of OAG risk factors. A familial aggregation study of risk factors for OAG suggested that optic disc signs may be stronger determinants of glaucoma in relatives than intraocular pressure (IOP). ${ }^{14}$

The frequency of $\beta$-PPA has been reported to increase with increasing levels of myopia. ${ }^{15}$ However, in these eyes, the etiology of the peripapillary morphology may not be uniform. Congenital anomalies of peripapillary tissue alignment and changes induced by scleral stretching may resemble age-related atrophic $\beta$-PPA. ${ }^{16-18}$ Myopia is also associated with OAG, ${ }^{19}$ suggesting that true atrophic $\beta$-PPA may occur as well.

OAG and myopia are both known to have genetic and environmental components, although the environmental component of neither is well understood. The relative genetic and environmental components of $\beta$-PPA and its relationship to both myopia and environmental factors are unknown. Such information may give insight into the relative contribution of inheritance in $\beta$-PPA and the relationship between $\beta$-PPA, myopia, and OAG. This study was conducted to analyze the relative importance of genes and environment on $\beta$-PPA in a large sample of adult twins.

\section{Methods}

\section{Subjects}

The study population consisted of 506 female twin pairs aged between 50 and 79 years, recruited from the Twins U.K. Adult Twin Registry, held at St. Thomas' Hospital in London. Registrants were ascertained from the general population of the United Kingdom through national media campaigns before the planning of any eye studies. Details of the registry have been described previously. ${ }^{20}$ Study subjects were examined between January 1998 and July 1999. Twelve pairs of twins were unable to attend for reasons of ill health or refusal to participate. Zygosity was determined in 506 twin pairs by standardized questionnaire $^{21}$ and confirmed by DNA short tandem repeat fingerprinting in approximately $40 \%$ of twin pairs in whom zygosity was uncertain. This procedure was used if there was any doubt about true zygosity or when the answers to the standardized questionnaire were not definitely monozygotic (MZ) or dizygotic (DZ).

\section{Measurements}

Examination included visual acuity testing using the ETDRS logMar (Early Treatment Diabetic Retinopathy Study logarithm of the minimum angle of resolution) chart and undilated refraction with an automatic refractor (Humphrey 670; Carl Zeiss Meditec, Dublin, CA). Spherical equivalent (SE) refraction was calculated in each eye. SE data were reproducible, and their distribution has been reported. ${ }^{22}$ After pupil dilatation, nonsimultaneous stereoscopic photographs centered 
on the optic nerve were taken of both eyes of all the twins. A camera (Kowa-Europe, Dusseldorf, Germany) was used with a $30^{\circ}$ field setting (Ektachrome 64 film; Eastman-Kodak, Rochester, NY). The same individual took all photographs, using the same camera, and the film was processed by the same laboratory. Photographs were examined using stereoscopic viewing spectacles on an $\mathrm{x}$-ray viewing light box. The best stereo pair was digitized with a slide scanner (Coolscan III; Nikon, Tokyo, Japan). Images of 1280 by 960 pixels were created at a magnification of $133 \%$.

\section{Grading of Peripapillary Atrophy}

The images were graded for the presence of peripapillary atrophy by one of two graders masked to the pairing and zygosity of subjects. The classification described by Jonas et al. ${ }^{7}$ was used to identify peripapillary atrophy. No deviations from this system were allowed. If present, $\beta$-PPA bordered the peripapillary scleral ring. It was characterized by visible large choroidal vessels and sclera, due to a denuded Bruch's membrane and loss of retinal pigment epithelium. It was distinguished from $\alpha$-PPA, characterized by irregular retinal hypo- or hyperpigmentation, which bordered zone beta or the scleral ring when the beta zone was absent. Examples of these two types of peripapillary atrophy are shown in Figure 1. $\beta$-PPA was graded as present, uncertain, absent, ungradable, or image missing and entered into an electronic database. Where graders disagreed or when an image was graded as uncertain, adjudication was made by one of the authors (PRH).

\section{Analytical Approach}

Data analysis was based on quantitative genetic modeling ${ }^{22,23}$ using the Mx program. ${ }^{24}$ In short, the technique is based on the comparison of the covariances (or correlations) within MZ and DZ twin pairs. Familial aggregation of a trait or disease suggests that genetic factors may be involved in etiology, but it does not exclude the possibility of shared environmental factors. Twin modeling assumes that MZ and DZ twin pairs share the same common (family) environment-the "equal environment" assumption-which has been tested and largely found to be true. ${ }^{25}$ As MZ pairs share the same segregating genes but DZ pairs share only half, any greater similarity between MZ pairs allows an estimation of this additional gene-sharing, using the equal-environment assumption. To quantify the genetic and environmental contributions to a dichotomous variable, such as the presence or absence of $\beta$-PPA, an underlying, continuous liability to $\beta$-PPA is assumed, which is affected by multiple genetic and environmental factors. ${ }^{26}$ Structural equation modeling uses variance-covariance matrix algebra to separate the observed phenotypic variance into additive (G) or dominant (D) genetic components and common (C) or unique (E) environmental components (E also includes measurement error). MZ twins share the same G genetic component, DZ twins 0.5, and MZ twins the D genetic component, whereas DZ twins share only 0.25 of $\mathrm{D}$, since there is a one-in-four chance that two siblings will share a dominant allele transmitted from one parent. Thus, the known twin-twin relationships were used in maximum likelihood methods to estimate the best-fitting model that fits the variance-covariance data obtained in the study. Dividing each of these components by the total variance yields the different standardized components of variance-for example, the heritability, which can be defined as the ratio of additive genetic variance to total phenotypic variance.

As $\beta$-PPA is a bivariate variable (yes/no) and refractive error is measured as a continuous variable (mean SE), to include both variables in a single model, polychoric correlations were estimated by using a saturated model, with refractive error divided into deciles to recode it

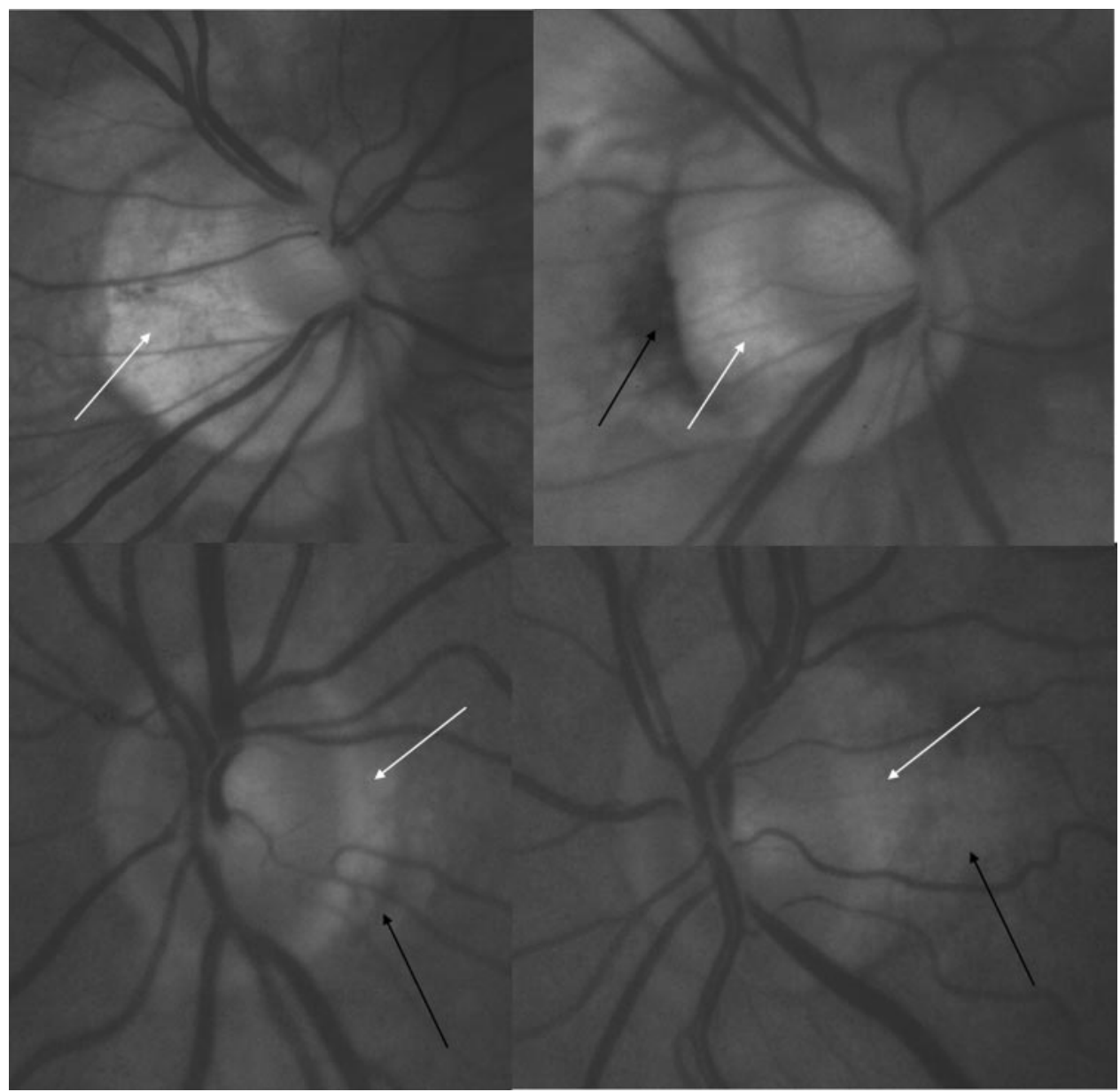

FIGURE 1. Peripapillary atrophy in monozygotic (top) and dizygotic (bottom) twin pairs. Black arrows: $\alpha$-PPA; white arrows: $\beta$-PPA 
Table 1. $\beta$-PPA Concordance in Twin Pairs

\begin{tabular}{lrcr}
\hline & $\boldsymbol{\beta}$-PPA & No PPA & Total \\
\hline MZ pairs & & & \\
$\beta$-PPA & 132 & 24 & 156 \\
No PPA & 14 & 30 & 44 \\
Total & 146 & 54 & 200 \\
DZ pairs & & 51 & \\
$\beta$-PPA & 137 & 28 & 62 \\
No PPA & 34 & 79 & 250 \\
Total & 171 & & \\
\hline
\end{tabular}

from a continuous measurement to a categorical one, allowing it to be modeled with the dichotomous $\beta$-PPA data. The univariate models were extended to include the bivariate cases of SE and $\beta$-PPA, to allow assessment of the extent to which any correlation between these two variables could be explained by common genes.

\section{Model Fitting Procedure}

Model fitting is used to try to explain the data in the most economic (parsimonious) manner. A backward-elimination procedure from the full model was used to develop a final model for the variance components $G, C$, and D in which the pattern of variances and covariances was explained by as few parameters as possible. Submodels were compared with the full model by hierarchic $\chi^{2}$ tests. The difference in $\chi^{2}$ values between submodel and full model is itself approximately distributed as a $\chi^{2}$, with degrees of freedom ( $d f$ ) equal to the difference in $d f$ of submodel and full model. Statistical software data handling and preliminary analyses were performed with commercial software (Stata Corp., College Station, TX). ${ }^{27}$ Research adhered to the tenets of the Declaration of Helsinki, and local ethics committee approval and informed consent were obtained.

\section{Results}

Optic disc photographs were gradable in 1813 eyes of 944 subjects (93.3\%) comprising $209 \mathrm{MZ}$ twin pairs and $263 \mathrm{DZ}$ twin pairs.

$\beta$-PPA was present in at least one eye of 254 subjects (25.1\%). The ocular prevalence was $22.9 \%$ in the right eyes and $21.7 \%$ in the left eyes. $\beta$-PPA was bilateral in 150 subjects or $59 \%$ of those with this sign. Figure 1 shows examples of $\beta$-PPA subtypes in MZ and DZ eyes. $\beta$-PPA prevalence did not vary significantly with zygosity (Tables 1 and 2 ).
Myopia was present in at least one eye of 221 (21.8\%) subjects. The prevalence of $\beta$-PPA increased with increasing myopia (Fig. 2). In left eyes, it increased from $17.9 \%$ in the nonmyopic eyes to $28.6 \%$ in low (SE, -1 to $-3 \mathrm{D}$ ), $40.5 \%$ in moderate (SE -2 to $-5 \mathrm{D}$ ), and $51.0 \%$ in high (SE, greater than $-5 \mathrm{D})$ myopia. In the right eyes, it increased from $18.5 \%$ in nonmyopic eyes to $34.9 \%$ in low (SE, -1 to $-3 \mathrm{D}$ ), $32.7 \%$ in moderate (SE -2 to $-5 \mathrm{D}$ ), and $57.5 \%$ in high (SE greater than -5 D) myopia.

Complete data for $200 \mathrm{MZ}$ and $250 \mathrm{DZ}$ twin pairs are shown in Table 2 . There were no statistically significant differences in prevalence of $\beta$-PPA or myopia between MZ and DZ twins. No statistically significant associations were found between age and presence of either $\beta$-PPA $(\beta=0.26, P=0.55)$ or myopia ( $\beta=-0.17, P=0.71$ ) in the linear regression analyses.

The polychoric correlation for $\beta$-PPA in right eyes was 0.76 (95\% CI, 0.57-0.88) for MZ pairs and 0.37 (95\% CI, 0.15-0.56) for $\mathrm{DZ}$ pairs, with the greater $\mathrm{MZ}$ correlation suggesting a genetic influence on the trait. Data for left eyes gave similar findings (MZ correlation, 0.76; DZ correlation, 0.34). Univariate modeling resulted in a best-fit model involving additive genetic effects and individual environment, with no shared environment or dominant genetic effect. The heritability of $\beta$-PPA in right eyes in univariate analysis was estimated as 0.75 for both right and left eyes.

Given the relationship between $\beta$-PPA and refractive error, as well as glaucoma, multivariate modeling to adjust for SE was performed. Using data for $\beta$-PPA in either eye (MZ correlation for $\beta$-PPA 0.72 ; DZ correlation, 0.28 ) and the mean SE for each individual divided into deciles (MZ/DZ correlations, $0.88 / 0.52$ ), the multivariate heritability for $\beta$-PPA was 0.70 (95\% CI, 0.53-0.83) and for mean SE, 0.88 (95\% CI, 0.850.91). There was an additive genetic correlation for the two phenotypes $(-0.21,95 \% \mathrm{CI},-0.33$ to -0.08$)$, suggesting some shared genes, as well as a significant nonshared environmental correlation $(-0.33,95 \% \mathrm{CI},-0.56$ to -0.06$)$. Genetic and environmental correlations are represented in Figure 3.

Multivariate modeling estimated that most of the genetic variance ( $67 \%$ of the overall $70 \%$ ) of $\beta$-PPA was due to genetic factors separate from refractive error and that only $3 \%$ of the genetic variance was due to shared genetic factors with mean SE (Fig. 4). Three percent of the variance was due to shared environmental factors with refractive error and $27 \%$ was due to specific unique environmental factors. The heritability of
FIGURE 2. The prevalence of $\beta$-PPA by degree of myopia in diopters (D) using SE refraction, for right and left eyes.

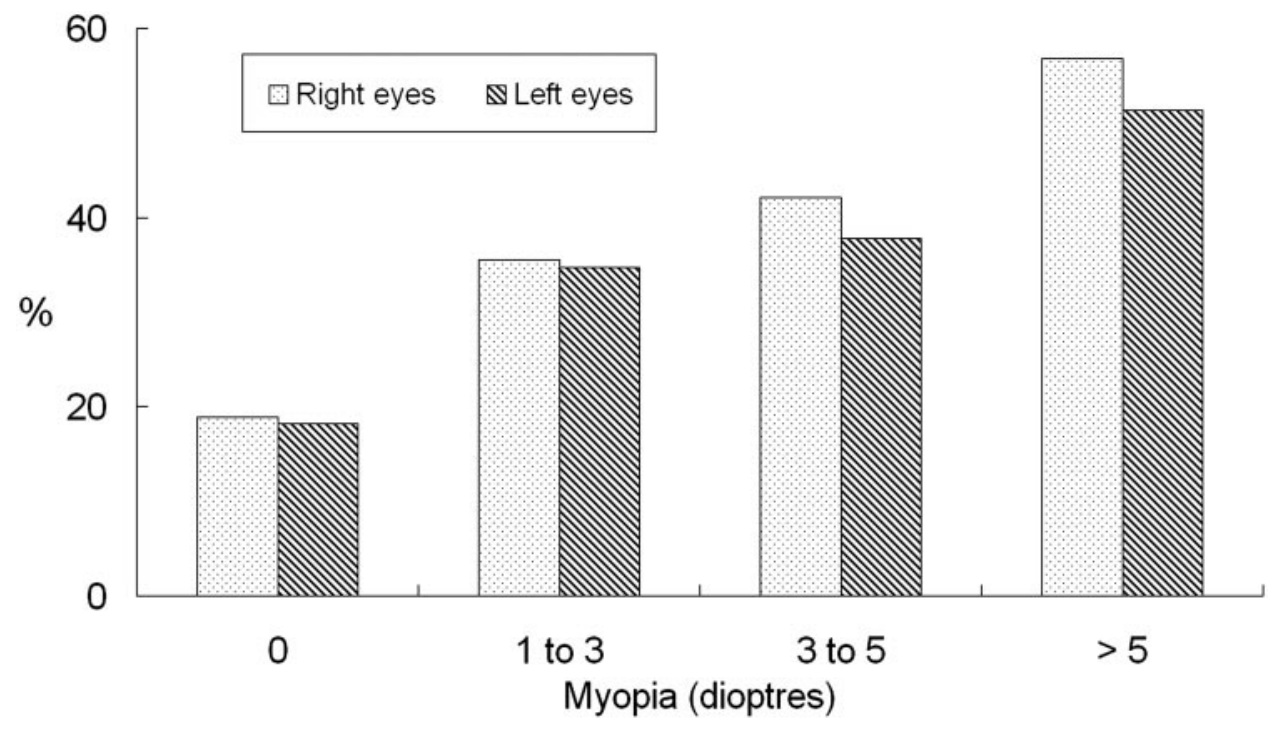


TABLE 2. $\beta$-PPA and Spherical Equivalent Refractive Error within MZ and DZ Twin Pairs

\begin{tabular}{lll}
\hline & \multicolumn{1}{c}{ MZ } & \multicolumn{1}{c}{$\mathbf{D Z}$} \\
\hline Number of pairs & 200 & 250 \\
$\beta$-PPA prevalence & 0.24 & 0.24 \\
Case-wise concordance (95\% CI) & $0.61(0.50-0.73)$ & $0.40(0.29-0.50)$ \\
Myopia (mean SE $<-0.5 \mathrm{D})$ & & \multicolumn{2}{l}{} \\
$\quad$ prevalence & 0.25 & 0.23 \\
Mean SE (D) & 0.38 & 0.41 \\
Correlation of SE & 0.89 & 0.49 \\
\hline
\end{tabular}

Data are for complete pairs.

$\beta$-PPA after adjustment for mean SE was 0.71 (95\% CI, $0.54-$ $0.84)$.

\section{Discussion}

The prevalence of $\beta$-PPA in this twin study is similar to that reported in singletons. In a very large study of normal subjects in Japan, $\beta$-PPA was found in $26 \%{ }^{28}$ Smaller clinic-based estimates of $\beta$-PPA frequency generally lie between $12 \%$ and $20 \%{ }^{1,7,29,30}$ This study confirms the relationship between $\beta$-PPA and myopia that has been reported in population-based studies, ${ }^{15,31}$ which may partially explain the differences in prevalence estimates between studies.

The similar prevalence of $\beta$-PPA in MZ and DZ twins suggests that there is no genetic association between $\beta$-PPA and being a member of a set of twins. These findings are in agree-

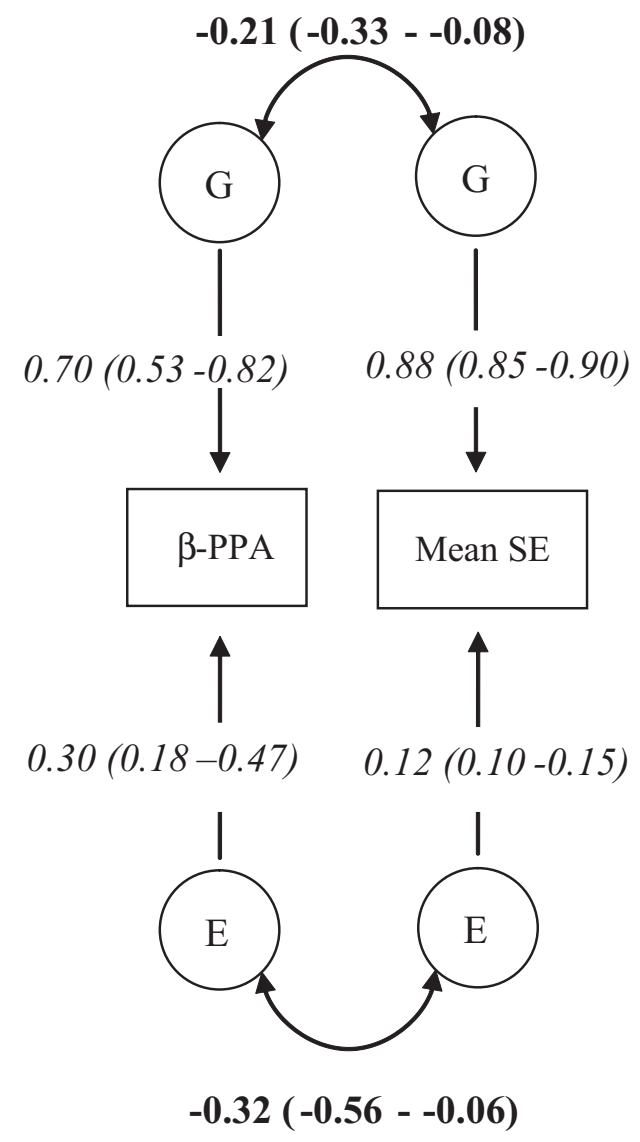

FIGURE 3. Genetic and environmental correlations (bold) and variance components (italic) of the best-fitting bivariate model for mean SE refraction and $\beta$-PPA. $\mathrm{G}$, additive genetic factor; $\mathrm{E}$, unique environmental factor.
$\square$ Specific genetic factors
$\square$ Unique environmental factors in common with Mean SE
$\square$ Genetic factors in common with Mean SE
- Specific unique environmental factors

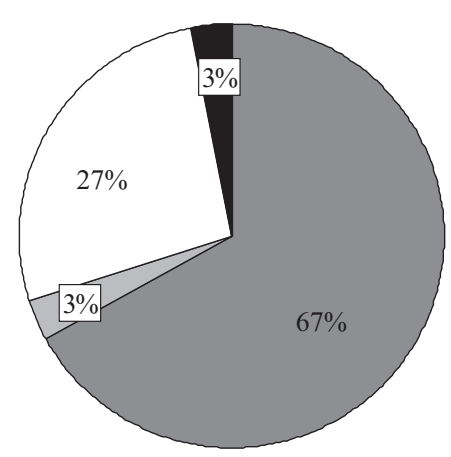

FIGURE 4. Sources of variance in $\beta$-PPA based on the best-fitting bivariate model.

ment with a systematic study of disease- and lifestyle-related characteristics between twins and singletons that suggests that generalization from twins to singleton populations is valid. ${ }^{32}$

Our data suggest that $\beta$-PPA has a strong heritable component. A heritability of $75 \%$ compares with $90 \%$ for myopia alone in this cohort, ${ }^{22} 58 \%$ for cortical cataract, ${ }^{33}$ and $45 \%$ for age-related maculopathy. ${ }^{34}$ Lower heritability of degenerative conditions such as ARM and cataract implies more population variation attributable to environmental factors. If one supposes $\beta$-PPA to be (as its name suggests) a feature caused by degeneration of the peripapillary tissues, then it is surprising that the heritability is closer to refractive error, which is generally occurs at a young age. It does not appear that common genes for myopia and $\beta$-PPA are the cause, as we estimated only 3\% of the genetic variance to have a common genetic basis. The explanation may relate to the possibility that $\beta$-PPA has several etiologies.

Two recent studies of children have suggested that in myopic eyes, peripapillary morphology may be partly congenital or acquired very early in life. One study reported a significant (34\%) prevalence of myopic peripapillary crescents in young Japanese children. In contrast, the prevalence of chorioretinal atrophy was only $16 \%{ }^{16}$ In the second study, Singaporean children were examined. It reported an association between increasing frequency of myopic temporal crescents and increasing myopic refraction and axial length but not an association with age. ${ }^{17}$ The hypothesis of a multifactorial etiology for myopic $\beta$-PPA is further strengthened by an angiographic study of myopic peripapillary morphology in adults. This study suggested that there may be two components to clinical $\beta$-PPA in these eyes: one related to stretching of the sclera and the other to vascular changes. ${ }^{18}$

Histologic studies of peripapillary atrophy in adults also suggest several distinct entities: simple misalignment of the choroid and RPE, malpositioning of the embryonic fold between the RPE and outer retina, and age-related atrophy of the peripapillary RPE and rods, usually surrounded by an area of RPE degeneration. ${ }^{35-38}$ In one study, atrophy was found in 15 of 17 otherwise normal eyes, suggesting that it may be a normal aging change. It varied from complete absence of the RPE to a loss of RPE pigmentation. ${ }^{35}$

It is not possible to distinguish clinically between these three histologic entities of peripapillary atrophy. A clinical distinction can be made between areas of severe or complete loss of RPE pigment and areas with irregular pigmentation. A peripapillary area through which sclera and large choroidal 
vessels can be seen is termed zone beta peripapillary atrophy. Areas of irregular hypo- or hyperpigmentation adjacent to the optic disc border or zone beta (when present) are termed zone alpha peripapillary atrophy. ${ }^{7}$

Clinically prevalent $\beta$-PPA is not nearly as common as histologic atrophy, even taking into account misclassification of congenital peripapillary misalignment. ${ }^{7}$ This disparity may be partly because the $\beta$-PPA is obscured by the overlying nerve fiber layer. ${ }^{35,39}$ Because there is no clear relationship between $\beta$-PPA defined clinically and true atrophy as defined histologically, one must presume a large potential for classification bias when using the clinical finding to classify the histologic state. The $\beta$-PPA, as ascertained in all clinical studies reported to date, may consist of both true atrophy and congenital malformations of the retinal layers. Were it possible to distinguish the two, the congenital variant of $\beta$-PPA might be highly heritable, whereas the atrophic variant may be more influenced by environmental factors. There may also be a difference between the relationships of each type of $\beta$-PPA with glaucoma. This hypothesis could be examined further by comparison of $\beta$-PPA heritability in younger adult and pediatric twin studies, as well as comparison with one of the several large studies of childhood refractive error, which are currently under way. ${ }^{40,41}$

\section{Conclusions}

This study provides the first information about the heritability of $\beta$-peripapillary atrophy and its relationship to refractive error. We estimate that the genetic effects are important with a heritability adjusted for refraction of $70 \%$. We confirmed an association with myopia but suggest that this not due to common genes. Rather, it is predominately due to the co-inheritance of genes specific for $\beta$-peripapillary atrophy. Although the relationship between the clinical sign and histologic phenotype needs further clarification, it raises the possibility of a genetic basis for optic disc morphologic characteristics associated with glaucoma.

\section{Acknowledgments}

The authors thank Mario Economou for assistance in digitizing the optic disc photographs.

\section{References}

1. Jonas JB, Nguyen XN, Gusek GC, Naumann GO. Parapapillary chorioretinal atrophy in normal and glaucoma eyes. I. Morphometric data. Invest Ophthalmol Vis Sci. 1989;30:908-918.

2. Elschnig A. Das colobom am sehnervenientritte und der conus nach unten. Graefes Arch Clin Exp Ophthalmol. 1900;51:391430.

3. Primrose J. Early signs of the glaucomatous disc. Br J Ophthalmol. 1971;55:820 - 825.

4. Wilensky JT, Kolker AE. Peripapillary changes in glaucoma. Am J Ophthalmol. 1976;81:341-345.

5. Anderson DR. Correlation of the peripapillary anatomy with the disc damage and field abnormalities in glaucoma. Doc Ophthalmol. 1983;35:1-10.

6. Heijl A, Samander C. Peripapillary atrophy and glaucomatous visual field defects. Doc Ophthalmol. 1985;42:403-407.

7. Jonas JB, Fernandez MC, Naumann GO. Glaucomatous parapapillary atrophy: occurrence and correlations. Arch Ophthalmol. 1992;110:214-222.

8. Budde WM, Jonas JB. Enlargement of parapapillary atrophy in follow-up of chronic open-angle glaucoma. Am J Ophthalmol. 2004; 137:646-654.

9. Jonas JB, Martus P, Budde WM, et al. Small neuroretinal rim and large parapapillary atrophy as predictive factors for progression of glaucomatous optic neuropathy. Ophthalmology. 2002;109:15611567.
10. Rockwood EJ, Anderson DR. Acquired peripapillary changes and progression in glaucoma. Graefes Arch Clin Exp Ophthalmol. 1998;226:510-515.

11. Uchida H, Ugurlu S, Caprioli J. Increasing peripapillary atrophy is associated with progressive glaucoma. Ophthalmology. 1998;105: 1541-1545.

12. Tezel G, Kolker AE, Kass MA, et al. Parapapillary chorioretinal atrophy in patients with ocular hypertension. I. An evaluation as a predictive factor for the development of glaucomatous damage. Arch Ophthalmol. 1997;115:1503-1508.

13. Tezel G, Kolker AE, Wax MB, et al. Parapapillary chorioretinal atrophy in patients with ocular hypertension. II. An evaluation of progressive changes. Arch Ophthalmol. 1997;115:1509-1514.

14. Wolfs RC, Klaver CC, Ramrattan RS, et al. Genetic risk of primary open-angle glaucoma: population-based familial aggregation study. Arch Ophthalmol. 1998;116:1640-1645.

15. Ramrattan RS, Wolfs RC, Jonas JB, et al. Determinants of optic disc characteristics in a general population: The Rotterdam Study. Ophthalmology. 1999;106:1588-1596.

16. Kobayashi K, Ohno-Matsui K, Kojima A, et al. Fundus characteristics of high myopia in children. Jpn J Ophthalmol. 2005;49:306311.

17. Tong L, Saw SM, Chua WH, et al. Optic disk and retinal characteristics in myopic children. Am J Ophthalmol. 2004;138:160-162.

18. Yasuzumi K, Ohno-Matsui K, Yoshida T, et al. Peripapillary crescent enlargement in highly myopic eyes evaluated by fluorescein and indocyanine green angiography. Br J Ophthalmol. 2003;87: 1088-1090.

19. Mitchell P, Hourihan F, Sandbach J, Wang JJ. The relationship between glaucoma and myopia: the Blue Mountains Eye Study. Ophthalmology. 1999;106:2010-2015.

20. Spector TD, Macgregor AJ. The St. Thomas' UK Adult Twin Registry. Twin Res. 2002;5:440 - 443.

21. Martin NG, Martin PG. The inheritance of scholastic abilities in a sample of twins. I. Ascertainments of the sample and diagnosis of zygosity. Ann Hum Genet. 1975;39:213-218.

22. Hammond CJ, Snieder H, Gilbert CE, Spector TD. Genes and environment in refractive error: the twin eye study. Invest $O p h$ thalmol Vis Sci. 2001;42:1232-1236.

23. Neale MC, Cardon LR. Methodology for Genetic Studies of Twins and Families. Dordrecht, The Netherlands: Kluwer Academic Publishers; 1992.

24. Neale MC. Mx Software. Richmond, Virginia: Department of Psychiatry, Medical College of Virginia; 1997.

25. Kyvik KO. Generalisability and assumptions in twin studies. In: Spector TD, Snieder H, Macgregor AJ, eds. Advances in Twin and Sib-Pair Analysis. London: Greenwich Medical Media; 2000:6778.

26. Falconer DS. Introduction to Quantitative Genetics. Harlow: Longman Scientific and Technical; 1989;112-186.

27. Stata Corp. STATA Statistical Software. ver. 7.0. College Station TX; 2001

28. Hayakawa T, Sugiyama K, Tomita G, et al. Correlation of the peripapillary atrophy area with optic disc cupping and disc hemorrhage. J Glaucoma. 1998;7:306-311.

29. Jonas JB, Gusek GC, Naumann GO. The parapapillary region of normal and glaucoma eyes. I Planimetric values of 312 glaucoma and 125 normal eyes (in German). Klin Monatsbl Augenheilkd. 1988; 193:52-61.

30. Jonas JB, Fernandez MC, Naumann GO. Parapapillary atrophy and retinal vessel diameter in nonglaucomatous optic nerve damage. Invest Ophthalmol Vis Sci. 1991;32:2942-2947.

31. Vongphanit J, Mitchell P, Wang JJ. Population prevalence of tilted optic disks and the relationship of this sign to refractive error. Am J Ophthalmol. 2002;133:679-685.

32. Andrew T, Hart DJ, Snieder H, et al. Are twins and singletons comparable? - a study of disease-related and lifestyle characteristics in adult women. Twin Res. 2001;4:464-477.

33. Hammond CJ, Duncan DD, Snieder H, et al. The heritability of age-related cortical cataract: the twin eye study. Invest Ophthalmol Vis Sci. 2001;42:601-605. 
34. Hammond CJ, Webster AR, Snieder H, et al. Genetic influence on early age-related maculopathy: a twin study. Opbthalmology. 2002; 109:730-736.

35. Curcio CA, Saunders PL, Younger PW, Malek G. Peripapillary chorioretinal atrophy: Bruch's membrane changes and photoreceptor loss. Ophthalmology. 2000;107:334-343.

36. Fantes FE, Anderson DR. Clinical histologic correlation of human peripapillary anatomy. Ophthalmology. 1989;96:20-25.

37. Jonas JB, Konigsreuther KA, Naumann GO. Optic disc histomorphometry in normal eyes and eyes with secondary angle-closure glaucoma. II. Parapapillary region. Graefes Arch Clin Exp Ophthalmol. 1992;230:134-139.
38. Kubota T, Jonas JB, Naumann GO. Direct clinico-histological correlation of parapapillary chorioretinal atrophy. $\mathrm{Br} \mathrm{J} \mathrm{Ophthalmol.}$ 1993;77:103-106.

39. Nevarez J, Rockwood EJ, Anderson DR. The configuration of peripapillary tissue in unilateral glaucoma. Arch Opbthalmol. 1988; 106:901-903.

40. Ojaimi E, Rose KA, Morgan IG, et al. Distribution of ocular biometric parameters and refraction in a population-based study of Australian children. Invest Opbthalmol Vis Sci. 2005;46:2748-2754.

41. Saw SM, Tong L, Chua WH, et al. Incidence and progression of myopia in Singaporean school children. Invest Opbthalmol Vis Sci. 2005;46:51-57. 